\title{
Controle de Aedes (Stegomyia) aegypti e Culex (Culex) quinquefasciatus Através de Formulados Contendo Bacillus thuringiensis israelensis em Temperaturas Controladas
}

\author{
João Antonio Cyrino Zequi ${ }^{\circledR}{ }^{凶}$, José Lopes $^{2}$ \& Fernando Pereira Santos²
}

1. Centro Universitário Filadélfia, e-mail: biologia@unifil.br (Autor para correspondência ${ }^{\bowtie}$ ), fernando.santos@unifil.br. 2. Universidade Estadual de Londrina, e-mail: jea@uel.br.

\author{
EntomoBrasilis 4 (3): 130-134 (2011)
}

Resumo. Aedes (Stegomyia) aegypti (Linnaeus) e Culex (Culex) quinquefasciatus Say são importantes vetores de patógenos em áreas urbanas. Este trabalho teve como objetivo avaliar diferentes formulados comerciais contendo Bacillus thuringiensis israelensis de Barjac, no controle de Ae. aegypti e $C x$. quinquefasciatus, verificando sua eficiência perante a potencia declarada. Testou-se os produtos Aquabac $®$ XT 1200 UTI/mg, Teknar $\circledR$

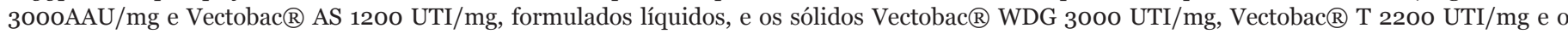
formulado experimental Biouel $500 \mathrm{UTI} / \mathrm{mg}$. Todos os produtos foram testados a temperatura de $25 \pm 2^{\circ} \mathrm{C}$ e os líquidos também a $15 \pm 2^{\circ} \mathrm{C}, 35 \pm 2^{\circ} \mathrm{C}$ e temperatura ambiente $\left(25,37\right.$ a $\left.28,73^{\circ} \mathrm{C}\right)$. Os testes foram realizados utilizando-se 25 larvas no início do $4^{\circ}$ estádio, em $150 \mathrm{~mL}$ de água destilada,

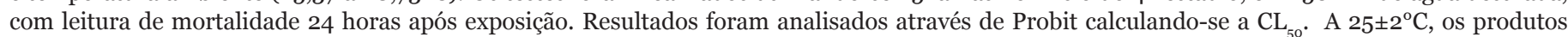
Vectobac WDG e Vectobac T foram os mais eficientes para Ae. aegypti, com $\mathrm{CL}_{50}$ respectivamente de 0,10 $(0,08-0,11) \mathrm{mg} / \mathrm{L}$ e $0,10(0,09-0,11$ $\mathrm{mg} / \mathrm{L}$ ). Para $C x$. quinquefasciatus, os mais eficientes foram Vectobac WDG, Vectobac AS, Biouel e Vectobac T. Tomando como referência a potência declarada dos produtos, o Biouel teve, proporcionalmente, melhor desempenho para as duas espécies. Nas temperaturas de $15 \pm 2^{\circ} \mathrm{C}, 35 \pm 2^{\circ} \mathrm{C}$ e na temperatura ambiente $\left(25,37\right.$ a $\left.28,37^{\circ} \mathrm{C}\right)$, Vectobac AS foi o mais eficiente para as duas espécies de Culicidae testadas. Na temperatura ambiente a $35 \pm 2^{\circ} \mathrm{C}$, necessitou-se de menor concentração dos produtos para controle de Cx. quinquefasciatus em relação à Ae. aegypti.

Palavras-Chave: Controle Biológico; Culicidae; Dengue; Vetor.

\section{Control of Aedes (Stegomyia) aegypti and Culex (Culex) quinquefasciatus Through Formulated Containing Bacillus thuringiensis israelensis at Controlled Temperatures}

Abstract. Aedes (Stegomyia) aegypti (Linnaeus) and Culex (Culex) quinquefasciatus Say are important pathogen vectors in urban environments. This study was designed to evaluate commercial formulations containing Bacillus thuringiensis israelensis de Barjac for the control of Ae. aegypti and $C x$. quinquefasciatus and to assess their efficiency as compared to manufacturers' claims. The tested products were the liquid formulation of

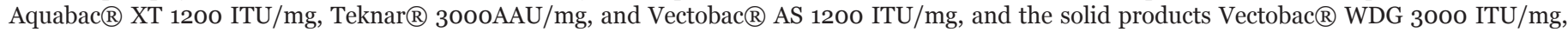
Vectobac ${ }^{\circledR}$ Tablet 2200 ITU/mg, and the trial formulation of Biouel 500 ITU/mg. All products were tested at $25 \pm 2^{\circ} \mathrm{C}$ temperature and the liquid formulations were also tested at $15 \pm 2^{\circ} \mathrm{C}, 35 \pm 2^{\circ} \mathrm{C}$, and at room temperature $\left(25.37\right.$ to $\left.28.73^{\circ} \mathrm{C}\right)$. The experiments were conducted with 25 larvae at the early 4 th stage, in $150 \mathrm{~mL}$ of distilled water; the dead larvae were counted 24 hours after product application. Results were analyzed using Probit to calculate $\mathrm{CL}_{50^{\circ}}$. The $25 \pm 2^{\circ} \mathrm{C}$ temperature, Vectobac WDG, and Vectobac Tablet were the most efficient in controlling Ae. aegypti, with $\mathrm{CL}_{50}$ of 0.10 $(0.08-0.11) \mathrm{mg} / \mathrm{L}$ and $0.10(0.09-0.11 \mathrm{mg} / \mathrm{L})$, respectively. The most efficient products for $C x$. quinquefasciatus were Vectobac WDG, Vectobac AS, Biouel, and Vectobac T. When the potency claimed by manufacturers was compared to our laboratory results, Biouel had the best performance for both species. Vectobac AS was the most efficient for both species of Culicidae tested at $15 \pm 2^{\circ} \mathrm{C}, 35 \pm 2^{\circ} \mathrm{C}$ and at room temperature (25.37 to $28.37^{\circ} \mathrm{C}$ ). Lower product concentrations were required at $35 \pm 2^{\circ} \mathrm{C}$ room temperature to control Cx. quinquefasciatus than for Ae. aegypti.

Keywords: Biological Control; Culicidae; Dengue; Vector.

$\boldsymbol{A}$ edes (Stegomyia) aegypti (Linnaeus) é o mosquito vetor do agente etiológico causador da febre dengue nos trópicos (Degallier et al. 2010). Segundo dados da Organização Mundial de Saúde, o dengue está presente em mais de 100 países e esta distribuição tem aumentado nas últimas décadas. Cerca de 2,5 bilhões de pessoas estão expostas ao risco de dengue e estima-se a ocorrência de mais de 50 milhões de casos da doença por ano no mundo. Em 2007, mais de 890.000 casos foram relatados nas Américas, sendo 26.00o casos de febre hemorrágica (WHO 2010).

Culex quinquefasciatus Say é um mosquito cosmopolita, domiciliado e era responsável por transmitir o nematóide da filariose em regiões como Manaus, Belém, Recife, Maceió e Salvador, dentre outras localidades do país (Deane 1951; Rachou 1956). Atualmente, apenas a região metropolitana de Recife, Pernambuco é considerada um área endêmica (Medeiros et al.
2003).

A filariose linfática expõe 905 milhões de pessoas ao risco de serem infectadas, sendo que 90 milhões de pessoas são afetadas mundialmente (MARGalit 1995). Segundo a OMS, até o final de 2007, 81 países estavam na lista de endêmicos para esta doença, com 750 milhões de pessoas em tratamento visando à erradicação da doença.

Uma das possibilidades eficiente e ecológica para o combate a esses vetores é o controle biológico utilizando-se o Bacillus thuringiensis israelensis de Barjac como princípio ativo de bioinseticidas comerciais, por sua atividade e especificidade sobre larvas de mosquitos e borrachudos (LACEY \& LACEY 1981; BECKER et al. 1992; Brown et al. 1998a, 1998b; RodRIGUes et al. 1999; Brown et al. 1999; Nayar et al. 1999; Brown et al. 2000; Chung et al. 2001; Filinger et al. 2003; GunaseKaran et al. 2004; Lopes et al. 2010; Bravo et al. 2011). 
Os diferentes tipos de formulados à base de $B$. thuringiensis israelensis são produzidos no estado físico líquido (suspensões aquosas ou concentrados emulsionáveis) ou sólido (pó, grânulos dispersíveis em água ou tabletes), visando à utilização em diferentes condições do criadouro e também na dependência da espécie alvo. Para o desenvolvimento de novos produtos, testes preliminares em laboratório são necessários para estimar sua possível eficiência nas condições de campo.

Este trabalho teve como objetivo avaliar diferentes formulados comerciais e um experimental, a base de $B$. thuringiensis israelensis, em condições de temperatura ambiente e controladas, sobre larvas de Ae. aegypti e Cx. quinquefasciatus, em experimentos de laboratório.

\section{MATERIAL E MÉTODOS}

Para a realização dos testes foram utilizadas larvas de Ae. aegypti e $C x$. quinquefasciatus do insetário contido no laboratório de Entomologia Geral e Médica da Universidade Estadual de Londrina. As criações tiveram início com a coleta de ovos a campo, utilizando-se armadilhas (ovitrampas) para Ae. aegypti e coletas manuais de jangadas de ovos de $C x$. quinquefasciatus em lagoas de tratamento de efluentes, com reposição mensal de material oriundo do campo.

Larvas de quarto estádio inicial eram utilizadas para os bioensaios, sendo que nenhum alimento era adicionado nas bandejas de criação 24 horas antes dos testes.

Foram testados os produtos comerciais Aquabac $\mathrm{R}$ XT $1200 \mathrm{UTI} / \mathrm{mg}$ (lote $\neq$ F295), Teknarß $3.000 A A U / m g$ (lote A206673); Vectobac $®$ AS $1200 \mathrm{UTI} / \mathrm{mg}$ (lote 69-149-N9), formulados líquidos, além dos formulados sólidos Vectobac $\mathbb{R}$ WDG $3000 \mathrm{UTI} / \mathrm{mg}$ (lote 72-72-578-PG), Vectobac $\AA$ T 2200 UTI/mg (lote PKoo9-2) e o formulado sólido produzido experimentalmente pelo Laboratório de Bioinseticida da Universidade Estadual de Londrina (Biouel - pó 500 UTI/mg), todos à base de B. thuringiensis israelensis.

Para padronização, Teknar foi transformado em UTI/ mg, pela fórmula UTI $=2,5 \times$ AAU, de acordo com (VILARINHos et al. 1998).

Os bioensaios para todos os produtos comparados foram realizados em condições de temperatura controlada de $25 \pm 2^{\circ} \mathrm{C}$ e para os três formulados líquidos Aquabac XT, Teknar e Vectobac AS, também em condições de temperaturas ambiente variável e controlada a $15 \pm 2{ }^{\circ} \mathrm{C}, 25 \pm 2{ }^{\circ} \mathrm{C}$ e $35 \pm 2^{\circ} \mathrm{C}$. Nos bioensaios em temperatura ambiente, utilizou-se termohigrômetro para mensurar os limites de temperatura.

A metodologia para os bioensaios foi baseada em LACEY (1997) e WHO $(1999,2005)$ com repetições em dias diferentes. Vinte e cinco larvas de $4^{\circ}$ estádio inicial eram transferidas para potes de polietileno circular transparente de $11 \mathrm{~cm}$ de diâmetro com $7 \mathrm{~cm}$ de profundidade, contendo $150 \mathrm{~mL}$ de água destilada. Os produtos foram avaliados em seis concentrações. Cada uma teve três replicas, e os testes foram repetidos 5 vezes em dias diferentes, totalizando 15 análises para cada produto.

A avaliação de mortalidade foi realizada 24 após a inoculação do bioinseticida e as pupas foram descontadas da estatística. Utilizou-se da análise de Probit (SPSS $₫$ 14.0 package

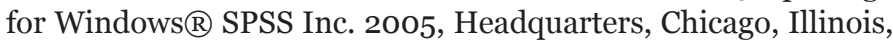
USA) para o bioensaio de resposta binária com o seguinte modelo matemático: $\mathrm{Pi}=\mathrm{F}(\alpha+\beta \mathrm{xi})$, onde $\mathrm{Pi}$ significa probabilidade da resposta; $x i=\log$ dose, $\alpha$ e $\beta=$ parâmetros e $F=$ função de distribuição acumulada (HADDAD 1998). Através do Probit determinou-se a concentração letal $\mathrm{CL}_{50}$ e seus intervalos de confiança, para comparar a eficiência dos produtos em relação as diversas temperaturas e UTI/mg dos produtos.

\section{RESULTADOS E DISCUSSÃO}

Nos bioensaios a $25 \pm 2{ }^{\circ} \mathrm{C}$, os formulados Vectobac T e WDG foram os mais eficientes para o controle de Ae. aegypti, com $\mathrm{CL}_{50}$ respectivamente de $0,10(0,09-0,11) \mathrm{mg} / \mathrm{L}$ e 0,10 (o,o8-0,11) mg/L, seguido de Teknar o,23 (0,21-0,25), Biouel 0,35 (0,31-0,40), Vectobac AS o,40 (0,37-0,45) e Aquabac 0,76 (o,62-1,06) (Tabela 1). Se levado em consideração a potência declarada dos produtos em UTI/mg, a qual deve se expressar em mortalidade larval, o Biouel é proporcionalmente mais eficiente em 1,7 e 1,3 vezes respectivamente quando comparado a WDG e Tablet, no controle de Ae. aegypti, já que sua potência declarada é de $500 \mathrm{UTI} / \mathrm{mg}$.

Para $C x$. quinquefasciatus, nos bioensaios a $25 \pm 2^{\circ} \mathrm{C}$, os melhores resultados foram obtidos com Vectobac WDG 0,09 (0,08-0,10), Vectobac AS 0,22 (0,21-0,24), Biouel 0,25 (0,230,27), Vectobac T o,31 (0,25-0,42), Teknar 0,39 (0,36-0,44) e Aquabac XT 0,47 $(0,42-0,56) \mathrm{mg} / \mathrm{L}$. Vectobac WDG mostrouse como o mais eficiente no controle das duas espécies testadas (Tabela 1); porém, também sobre esta espécie, o Biouel, levandose em consideração a quantidade de UTI/ mg utilizada para atingir a $\mathrm{CL}_{50}$, teve o melhor desempenho, mostrando eficiência e eficácia contra estas duas espécies de mosquitos.

Para Vectobac T, além da sua eficiência sobreas larvas dos culicídeos testados (Tabela 1), a sua formulação em comprimido poderia facilitar a operação em campo, caracterizando-o como apropriado para controle de Ae. aegypti, podendo ser facilmente manipulado em estratégias de controle quando o criadouro for de difícil acesso. O produto experimental Biouel mostrou-se como alternativa viável de controle para esses mosquitos, com resultados compatíveis aos formulados importados (Tabela 1). Aquabac XT necessitou de uma maior concentração para atingir a $\mathrm{CL}_{50}$ nas duas espécies de Culicidae testadas em todas as temperaturas (Tabela 1 ).

O produto Vectobac WDG mostrou ação semelhante a $25 \pm 2{ }^{\circ} \mathrm{C}$ para Ae. aegypti e $C x$. quinquefasciatus (Tabela1), enquanto que Vectobac $\mathrm{T}$ e Teknar necessitaram de uma concentração maior para atingir a $\mathrm{CL}_{50}$ em imaturos de $C x$. quinquefasciatus, em relação aos resultados obtidos para Ae. aegypti. Com os produtos na formulação líquida, nas temperaturas de 25,4 a $28,7^{\circ} \mathrm{C}$ e a $35 \pm 2^{\circ} \mathrm{C}$, necessitou-se de menor concentração dos produtos para controle de $C x$. quinquefasciatus em relação a Ae. aegypti. Mulla (1990) afirma que entre os Culicidae existem diferentes níveis de suscetibilidade para o $B$. thuringiensis israelensis, sendo que em geral, Culex são mais suscetíveis, Aedes são menos e Anopheles são tolerantes a maioria dos formulados de $B$. thuringiensis israelensis. Os resultados obtidos corroboram com as afirmações de MulLA (1990). Ruas-Neto et al. (1994) encontraram uma $\mathrm{CL}_{50}$ de 1,15 e 1,09 (log-probit) respectivamente para os produtos Vectobac AS e Teknar em testes de laboratórios com $C x$. quinquefasciatus. AmalRAJ et al. (2000), testando Vectobac AS em condições de laboratórios, constataram que o formulado foi relativamente mais efetivo sobre $C x$. quinquefasciatus do que sobre Ae. aegypti e Anopheles stephensi Liston, 1901, com respectivos valores de $\mathrm{CL}_{50}$ de 0,046 , o,06o e 0,190 mg/L, comprovando uma menor susceptibilidade de Ae. aegypti ao produto quando comparado a $C x$. quinquefasciatus. LOPEs et al. (2010) também encontrou uma alta sensibilidade de $C x$. quinquefasciatus em relação a Ae. aegypti testando Biouel, formulado liquido contendo $B$. thuringiensis israelensis.

A eficiência de $B$. thuringiensis israelensis depende do tipo de formulação, hábitos alimentares das espécies de Culicidae e suscetibilidade dos insetos (LOPEs et al. 2010). Os resultados obtidos neste experimento, onde Vectobac T e Teknar a $25 \pm 2{ }^{\circ} \mathrm{C}$ apresenta melhor desempenho sobre Ae. aegypti, em comparação a $C x$. quinquefasciatus diferencia-se dos dados acima discutidos, e seleciona estes formulados como mais eficientes para o controle de Ae. aegypti, nesta condição.

Baseado nos testes de laboratórios, os formulados promissores para testes em campo no controle de Ae. aegypti são: Vectobac T, Vectobac WDG e Biouel, já que são formulados sólidos e de fácil aplicação em locais de procriação desse mosquito. Para $C x$. quinquefasciatus, mosquito típico de ambiente poluído, os produtos que poderiam ser promissores,, baseado nos testes em laboratórios e a facilidade de aplicação no criadouro, são 
Tabela 1. UTI(s)/mg utilizada para atingir a $\mathrm{CL}_{50}$ e seus limites, para diferentes formulados comerciais com Bacillus thuringiensis israelensis sobre larvas de $4^{\circ}$ instar inicial de Aedes aegypti e Culex quinquefasciatus, em diferentes condições de temperaturas e fotoperíodo de 12L:12E.

\begin{tabular}{|c|c|c|c|c|}
\hline $\begin{array}{r}\text { Produto } \\
\left(25 \pm 2^{\circ} \mathrm{C}\right) \\
\end{array}$ & $\begin{array}{c}\text { Ae. aegypti } \\
\mathrm{CL}_{50} \text { (mg/L) (Max.-Min.) }\end{array}$ & UTI/mg & $\begin{array}{l}\text { Cx. quinquefasciatus } \\
\mathbf{C L}_{50} \text { (mg/L) (Max.-Min.) }\end{array}$ & UTI/mg \\
\hline Aquabac XT & $0,76(0,62-1,06)$ & 912 & $0,47(0,42-0,56)$ & 564 \\
\hline Teknar & $0,23(0,21-0,25)$ & 1725 & $0,39(0,36-0,44)$ & 1170 \\
\hline Vectobac AS & $0,40(0,37-0,45)$ & 480 & $0,22(0,21-0,24)$ & 264 \\
\hline Vectobac T & $0,10(0,09-0,11)$ & 220 & $0,31(0,25-0,42)$ & 682 \\
\hline Vectobac WDG & $0,10(0,08-0,11)$ & 300 & $0,09(0,08-0,10)$ & 270 \\
\hline Biouel & $0,35(0,31-0,40)$ & 175 & $0,25(0,23-0,27)$ & 125 \\
\hline $\begin{array}{l}\text { Produto } \\
\left(15 \pm 2^{\circ} \mathrm{C}\right)\end{array}$ & $\begin{array}{c}\text { Ae. aegypti } \\
\mathrm{CL}_{50} \text { (mg/L) (Max.-Min.) }\end{array}$ & UTI/mg & $\begin{array}{l}\text { Cx. quinquefasciatus } \\
\mathrm{CL}_{50} \text { (mg/L) (Max.-Min.) }\end{array}$ & UTI/mg \\
\hline Aquabac XT & $0,66\left(0,55^{-0}, 85\right)$ & 792 & $0,58(0,48-0,83)$ & 696 \\
\hline Teknar & $0,27(0,22-0,40)$ & 810 & $0,49(0,43-0,57)$ & 1470 \\
\hline Vectobac AS & $0,24(0,22-0,25)$ & 288 & $0,43(0,39-0,50)$ & 516 \\
\hline $\begin{array}{l}\text { Produto } \\
\left(35 \pm 2^{\circ} \mathrm{C}\right)\end{array}$ & $\begin{array}{c}\text { Ae. aegypti } \\
\mathrm{CL}_{50}(\mathrm{mg} / \mathrm{L})(\text { Max.-Min.) }\end{array}$ & UTI/mg & $\begin{array}{l}\text { Cx. quinquefasciatus } \\
\mathrm{CL}_{50}(\mathrm{mg} / \mathrm{L}) \text { (Max.-Min.) }\end{array}$ & UTI/mg \\
\hline Aquabac XT & $0,70(0,59-0,90)$ & 840 & $0,28(0,23-0,35)$ & 336 \\
\hline Teknar & $0,32(0,26-0,45)$ & 960 & $0,20(0,18-0,23)$ & 600 \\
\hline Vectobac AS & $0,30(0,28-0,33)$ & 360 & $0,10(0,08-0,11)$ & 120 \\
\hline $\begin{array}{c}\text { Produto } \\
\left(25,4 \text { a } 28,7^{\circ} \mathrm{C}\right)\end{array}$ & $\begin{array}{c}\text { Ae. aegypti } \\
\mathrm{CL}_{50}(\mathrm{mg} / \mathrm{L}) \text { (Max.-Min.) }\end{array}$ & UTI/mg & $\begin{array}{l}\text { Cx. quinquefasciatus } \\
\mathrm{CL}_{50} \text { (mg/L) (Max.-Min.) }\end{array}$ & UTI/mg \\
\hline Aquabac XT & $0,49(0,45-0,54)$ & 588 & $0,27(0,24-0,29)$ & 324 \\
\hline Teknar & $0,36(0,27-0,64)$ & 1080 & $0,21(0,20-0,22)$ & 630 \\
\hline Vectobac AS & $0,28(0,24-0,33)$ & 336 & $0,19(0,18-0,20)$ & 228 \\
\hline
\end{tabular}

Vectobac WDG, Vectobac AS e Teknar (Tabela 1), necessitando portanto de testes a campo. Os sólidos como Biouel e Vectobac $\mathrm{T}$ apresentaram resultados compatíveis, mas com formulação que possa apresentar dificuldades operacionais nesse tipo de ambiente, sendo então indicada para se testar a campo em pequenos criadouros.

A $35 \pm 2{ }^{\circ} \mathrm{C}$, houve diminuição da $\mathrm{CL}_{50}$ em relação à temperatura de $25 \pm 2^{\circ} \mathrm{C}$ para os três produtos testados em $C x$. quinquefasciatus e Ae. aegypti, com exceção para Teknar em relação a esta última espécie (Tabela 1). Este resultado pode estar diretamente relacionado à atividade alimentar e ao metabolismo da larva, que pode acelerar com o aumento na temperatura, mas, provavelmente esta variação na temperatura não interfere na estrutura do cristal protéico.

A faixa de variação da temperatura ambiente do laboratório esteve mais próxima às condições de temperatura de $25 \pm 2^{\circ} \mathrm{C}$, todavia os resultados de $\mathrm{CL}_{50}$ não acompanharam os resultados nos testes com Ae. aegypti. O Aquabac XT teve a menor $\mathrm{CL}_{50}$ obtida em todos os testes tanto para Ae. aegypti como para ${ }^{50} x$. quinquefasciatus (Tabela 1 ). Para Teknar que havia apresentado menor $\mathrm{CL}_{50}$ a $25 \pm 2^{\circ} \mathrm{C}$, o resultado foi superior ao obtido nos bioensaios a $35 \pm 2{ }^{\circ} \mathrm{C}$. Para Vectobac AS houve diminuição da $\mathrm{CL}_{50}$, estando mais próximo ao obtido a $35 \pm 2{ }^{\circ} \mathrm{C}$ (Tabela 1). Em $C x$. quinquefasciatus, os resultados foram mais uniformes, onde Aquabac XT e Teknar tiveram resultados mais próximos à condição de $35^{\circ} \mathrm{C}$, e somente o Vectobac $\mathrm{AS}$ mostrou semelhança ao obtido para $25 \pm 2^{\circ} \mathrm{C}$. De forma geral, Vectobac AS nas temperaturas de $15 \pm 2^{\circ} \mathrm{C}, 35^{ \pm 2}{ }^{\circ} \mathrm{C}$ e 25,37 a $28,37^{\circ} \mathrm{C}$ foi o mais eficiente para as duas espécies de Culicidae testadas (Tabela 1).

A falta de uniformidade nos resultados obtidos para Ae. aegypti pode estar relacionada à menor suscetibilidade da larva desta espécie e também a diferenças individuais dentro da população. Nesse contexto, a temperatura interfere indiretamente na ação do $B$. thuringiensis israelensis, conforme relatado em LACEY \& UndeEN (1984), KATBEH-BAder (1999), NAyAR et al. (1999). BECKER et al. (1992), que encontraram diferença na eficácia de $B$. thuringiensis israelensis especialmente entre $5^{\circ}$ e $8^{\circ} \mathrm{C}$ em $2^{\circ}$ e $4^{\circ}$ instares de Aedes vexans (Meigen 1830), provavelmente pela redução da filtração das larvas em baixas temperaturas, sugerindo que se deve aplicar grande quantidade de produto abaixo de $8^{\circ} \mathrm{C}$ para esta espécie. Culex saltanensis, exposto a $B$. thuringiensis israelensis na temperatura de $12 \pm 1^{\circ} \mathrm{C}$, e fotoperíodo $14 \mathrm{~L}: 10 \mathrm{E}$, teve sua suscetibilidade diminuída em 1,5 vezes em relação a $\mathrm{CL}_{50}$, quando comparado com a temperatura ambiente (ZEQUI \& LOPES 2007).

$\mathrm{Na}$ análise dos resultados para $C x$. quinquefasciatus é possível detectar diferenças da $\mathrm{CL}_{50}$ nas faixas de temperatura empregadas, onde necessitou-se de maior concentração de produto a $15 \pm 2{ }^{\circ} \mathrm{C}$ em relação a $35 \pm 2^{\circ} \mathrm{C}$, compatível ao observado por NAYAR et al. (1999), que encontraram uma $\mathrm{CL}_{50}$ respectivamente de $0,152,0,139$ e $0,140 \mathrm{mg} / \mathrm{L}$ em bioensaios mantidos a $15^{\circ} \mathrm{C}, 25^{\circ} \mathrm{C}$ e $35^{\circ} \mathrm{C}$, para $4^{\circ}$ instar de $C x$. nigripalpus submetidos à Vectobac $\AA 12 \mathrm{AS}-1.200 \mathrm{Uti} / \mathrm{mg}$, em 24 horas. KATBEH-BADER et al. (1999), testando os produtos padrões de B. thuringiensis israelensis (IPS-82) e Bacillus sphaericus Neide (SPH88 2362) sobre Culiseta longiareolata (Macquart), verificaram que a mortalidade aumenta significativamente com o aumento da temperatura, onde foram menos suscetíveis a $2 \mathrm{O} \pm 1^{\circ} \mathrm{C}$, do que a altas temperaturas de $25 \pm 1^{\circ} \mathrm{C}$ e $28 \pm 1^{\circ} \mathrm{C}$.

A temperatura aumenta a taxa de alimentação dos imaturos, com conseqüente aumento de ingestão de B. thuringiensis israelensis. Assim, a temperatura afeta indiretamente a ação de $B$. thuringiensis israelensis, já que em temperaturas baixas, ocorre menor filtração dos imaturos, necessitando aplicação de uma concentração maior de produto para se obter resultados satisfatórios (BECKER et al. 1992).

Conclui-se que todos os formulados foram eficientes para o controle de larvas de Ae. aegypti e Cx. quinquefasciatus nas condições experimentais empregadas. Entre os formulados testados, Vectobac T, Vectobac WDG e o Biouel são os mais indicados para o controle de Ae. aegypti, e Vectobac AS e WDG para o controle de $C x$. quinquefasciatus em temperatura de $25 \pm 2^{\circ} \mathrm{C}$. Vectobac AS mostrou melhor eficiência para Ae. aegypti e $C x$. quinquefasciatus nas temperaturas de $15 \pm 2^{\circ} \mathrm{C}, 35 \pm 2{ }^{\circ} \mathrm{C}$ e variável de 25,37 a $28,37^{\circ} \mathrm{C}$. 


\section{AGRADECIMENTOS}

A Fundação Araucária (Apoio ao Desenvolvimento Científico e Tecnológico do Paraná) e ao Programa de Pós-graduação em Agronomia da Universidade Estadual de Londrina.

\section{REFERÊNCIAS}

Amalraj, D.D., S.S. Sahu, P. Jambulingam, D.O.S.S. Boopathi, M. Kalyanasundaram, \& P.K. Das, 2000. Efficacy of aqueous suspension and granular formulations of Bacillus thuringiensis (Vectobac) against mosquito vectors. Acta Tropica, 75: 243-246.

Becker, N., M. Zgomba, M. Ludwig, D. Petric \& F. Rettich, 1992. Factors influencing the activity of Bacillus thuringiensis var. israelensis treatments. Journal of the American Mosquito Control Association, 8: 285-289.

Bravo, A., S. Likitvivatanavong, S.S. Gill \& M. Soberón, 2011. Bacillus thuringiensis: A story of a successful bioinsecticide. Insect Biochemistry and Molecular Biology, 41: 423-431.

Brown, M.D., T. Darran, M. Paul, G.G. Jack \& H.K. Brian, 1999. Laboratory and field evaluation of the efficacy of four insecticides for Aedes vigilax (Diptera: Culicidae) and toxicity to the nontarget shrimp Leander tenuicornis (Decapoda: Palaemonidae). Journal Economic Entomologist, 92: 1045 $-1051$.

Brown, M.D., T. Darran \& H. K. Brian, 1998a. Laboratory and field evaluation of efficacy of Vectobac $\AA 12$ AS against Culex sitiens (Diptera: Culicidae) larvae. Journal of the American Mosquito Control Association, 14: $183-185$.

Brown, M.D., T. Darran \& H.K. Brian, 1998b. Acute toxicity of selected pesticides to the pacific blue - eye, Pseudomugil signifer (Pisces). Journal of the American Mosquito Control Association, 14: $463-466$.

Brown, M.D., M.W. Tonya, G. Susannah, G.G. Jack, P. David \& H.K. Brian, 2000. Toxicity of insecticides for control of freshwater Culex annulirostris (Diptera: Culicidae) to the nontarget shrimp, Caradina indistincta (Decapoda: Atyidae). Journal Economic Entomologist, 93: 667 - 672.

Chung, Y.K., S.G. Phua-Lam, Y.T. Chua \& R. Yatiman, 2001. Evaluation of biological and chemical inseticide mixture against Aedes aegypti larvae and adults by thermal fogging in Singapore. Medical and Veterinary Entomology, 15: 321-327.

Deane, L.M, 1951. Observações sobre alguns hábitos dos adultos de Culex fatigans, o principal transmissor da filariose em Belém, Pará. Revista do Serviço Especial de Saúde Pública, 4, $423-464$.

Degallier, N., C. Favier, C. Menkes, M. Lengaigne, W. M. Ramalho, R. Souza, J. Servain \& J. P. Boulanger. 2010. Toward an early warning system for dengue prevention: modeling climate impact on dengue transmission. Climatic Change, 98: 581-592.

Filinger, U., B.G.J. Knols \&N. Becker, 2003. Efficacy and efficiency of new Bacillus thuringiensis var. israelensis and Bacillus sphaericus formulations against Afrotropical anophelines in Western Kenya. Tropical Medicine and International Health, 8: $37-47$.

Gunasekaran, K., P.S. Boopathi Doss, K. Vaidyanathan. 2004. Laboratory and field evaluation of Teknar HP-D, a biolarvicidal formulation of Bacillus thuringiensis ssp. israelensis, against mosquito vectors. Acta Tropica, 92: 109-118.

Haddad, M.L., 1998. Utilização do Polo-PC para análise de Probit. In: Alves, S.B. Controle Microbiano de Insetos, Piracicaba, FEALQ, 1013p.

Katbch-Bader, A., H. Khyami-Horani \& Z.H. Mohsen, 1999. Effect of temperature on the susceptibility of Culiseta longiareolata (Macquart) (Dipt. Culicidae) to two standard strains of biocontrol bacteria. Journal of Applayed Entomology, 123: 629-631.

Lacey, L.A., 1997. Laboratory biossay of bacteria against aquatic insects with emphasis on larvae of mosquitoes and black flies. p. 79-9o. In: Lacey, L.A. Manual of Techniques in Insect Pathology, London, Academic Press, 409p.

Lacey, L.A. \& J.M. Lacey, 1981. The larvicidal activity of Bacillus thuringiensis var. israelensis $(\mathrm{H}-14)$ against mosquitoes of the central Amazon basin. Mosquitoes. News. 41: 266 - 270.

Lacey, L.A. \& A.H. Undeen, 1984. The effect of formulation, concentration, and application time on the efficacy of Bacillus thuringiensis (H14) against black fly larvae under natural conditions. Journal Economic Entomologist. 77: 412-418.

Lopes J., O.M.N. Arantes \& M.A. Cenci. 2010. Evaluation of a new formulation of Bacillus thuringiensis israelensis. Brazilian Journal of Biology. 70: 1109-1113.

Lozovei, A.C., 2001. Culicídeos (mosquitos). p. 59-103. In Marcondes, C.B. Entomologia Médica e Veterinária, São Paulo: Editora Atheneu, 432p.

Manasherob, R., E. Bem-Dov, J. Margalit, A. Zaritsky \& Z. Barak, 1997. Raising activity of Bacillus thuringiensis var. israelensis against Anopheles stephensi larvae by encapsulation in Tetrahymena pyriformis (Hymenostomatida: Tetrahymenidae) Journal of the American Mosquito Control Association. 12: 627-631.

Margalit, J., N. Becker, C. Back \& A. Zaritsky, 1995. Bacillus thuringiensis subsp. israelensis as a Biological Control Agent of Mosquitoes and Black Flies. p. 521-556. In: Feng, T.Y.; K.F. Chak, A.R. Smith, J. Margalit, C. Chilcott, \& R.I. Rose. Bacillus thuringiensis Biotechnology and Environmental Benefits. 1, Hua Shiang Yuan Publishing Co. Taipei, Taiwan, $525 \mathrm{p}$.

Medeiros, Z., E.P. Cesse., J.A. Menezes \& F. Lessa. 2003. Controle da filariose linfática no Brasil, 1951-200o. Epidemiologia e Serviços de Saúde 12: 77-86.

Mulla, M.S., 1990. Activity, field efficacy, and use of Bacillus thuringiensis israelensis against mosquitoes. p. 134-160. In Barjac, H. \& D. Sutherland. Bacterial control of mosquitoes e black flies. New Brunswick: Rulegers University Press, 349p.

Nayar, J.R., J.W. Knight, D.B. Carlson \& P.D. O'bryan, 1999. Laboratory evaluation of biotic and abiotic factors that may influence larvicidal activity of Bacillus thuringiensis serovar israelensis against two Florida mosquitoes species. Journal of the American Mosquito Control Association. 15: 32-42.

Rachou, R.G., 1956. Transmissores da filariose bracroftiana no Brasil. Revista Brasileira de Malariologia e Doenças Tropicais. 8: $267-279$.

Rodrigues, I.B., P.T. Wanderli \& M.C.S.D. José, 1998. Studies on the Bacillus sphaericus larvicidal activity against Malarial Vector Species in Amazonia. Memórias do Instituto Oswaldo Cruz. 93: $441-444$.

Ruas-Neto, A.L., S.M. Silveira, E.R.C. Colares, 1994. Mosquito control base on larvicides in the state of Rio Grande do Sul, Brazil choice of the control agent. Caderno de Saúde Pública. Rio de Janeiro, 10: 222-230.

Tauil, P.L., 2002. Aspectos críticos do controle do dengue no Brasil. Caderno Saúde Pública, 18: 867-71.

World Health Organization. 1999. DRAFT. Determination of the Toxicity of Bacillus thuringiensis subsp. israelensis and B. sphaericus products, p.29-33. In: Who/Cds/Cpc/ Whopes/99.2. Guideline specifications for bacterial larvicides for public healt use. 33p.

World Health Organization. 2005. Guidelines for Laboratory an Field Testing of Mosquito Larvicides. p.03-36. Disponível em: <http://whqlibdoc.who.int/hq/2005/WHO CDS WHOPES GCDPP 2005.13.pdf > acesso em 29/06/2010.

World Health Organization. 2010. Impact of dengue, 1: 1. Disponível em: < http://www.who.int/csr/disease/dengue/ impact/en/> acesso em 29/06/10.

Vilarinhos, P.T.R., J.M.C.S. Dias, C.F.S. Andrade \& C.J.P.C. Araújo-Coutinho, 1998. Uso de bactérias para o controle de culicídeos e simulídeos, p. 447-480. In: Alves, S.B. (Editor). Controle Microbiano de Insetos, FEALQ, 1163p.

Zequi, J.A.C \& J. Lopes, 2007. Biological control of Culex (Culex) saltanensis Dyar, (Diptera, Culicidae) through Bacillus 
thuringiensis israelensis in laboratory and field conditions. Revista Brasileira de Zoologia, 24 (1): 164-168.

\section{Como citar este artigo:}

Zequi, J.A.C., J. Lopes \& F.P. Santos, 2011. Controle de Aedes (Stegomyia) aegypti e Culex (Culex) quinquefasciatus através de formulados contendo Bacillus thuringiensis israelensis em temperaturas controladas. EntomoBrasilis, 4(3): 130-134. www.periodico.ebras.bio.br/ojs
Recebido em: 18/03/2011

Aceito em: 11/06/2011

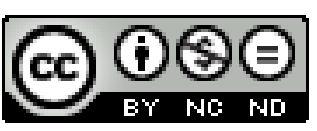

Entomo Breasing

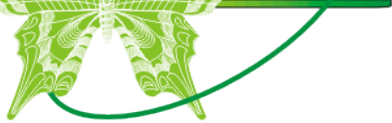

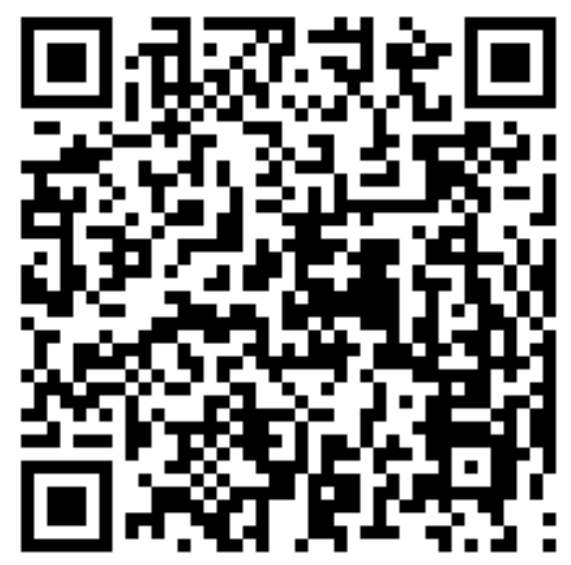

